\title{
Trivium
}

Revue franco-allemande de sciences humaines et sociales - Deutsch-französische Zeitschrift für Geistesund Sozialwissenschaften

29 | 2019

Regards croisés sur le prophète de l'Islam

\section{Regards croisés sur le prophète de l'Islam}

Introduction

\section{Francesco Chiabotti et Ruggero Vimercati Sanseverino}

\section{OpenEdition}

\section{Journals}

Édition électronique

URL : http://journals.openedition.org/trivium/6309

DOI : $10.4000 /$ trivium.6309

ISBN : 1963-1820

ISSN : 1963-1820

Éditeur

Les éditions de la Maison des sciences de l'Homme

Référence électronique

Francesco Chiabotti et Ruggero Vimercati Sanseverino, "Regards croisés sur le prophète de l'Islam », Trivium [En ligne], 29 | 2019, mis en ligne le 17 octobre 2019, consulté le 08 septembre 2020. URL: http://journals.openedition.org/trivium/6309; DOI : https://doi.org/10.4000/trivium.6309

Ce document a été généré automatiquement le 8 septembre 2020.

\section{cc)}

Les contenus des la revue Trivium sont mis à disposition selon les termes de la Licence Creative Commons Attribution - Pas d'Utilisation Commerciale - Pas de Modification 4.0 International. 


\title{
Regards croisés sur le prophète de l'Islam
}

\author{
Introduction
}

Francesco Chiabotti et Ruggero Vimercati Sanseverino

1 L'idée de présenter un dossier sur le prophète de l'islam est née au cours de la première année du projet franco-allemand ANR-DFG «PROPHET - Muhammad in the Mirror of his community in early modern and modern islam $»^{1}$. Cette vaste enquête, qui a occupé un grand nombre de chercheurs issus de spécialisations très différentes (tant au niveau des aires géographiques que des disciplines) a pour vocation de mettre en relation les spécificités françaises et allemandes de la recherche sur l'islam ${ }^{2}$. Les deux pays possèdent des traditions importantes d'études historiques et littéraires sur l'islam. Dès la naissance des disciplines dites «orientalistes", les contacts tantôt féconds, parfois conflictuels, furent au cœur de « l'émergence de cette nouvelle discipline que l'on peut qualifier d'européenne», selon la formulation de Michel Espagne ${ }^{3}$. La nécessité d'un travail commun est à l'origine de la constitution d'un groupe de recherche qui s'est impliqué dans une vaste exploration de la réception de la figure prophétique de l'islam dans les périodes prémoderne, moderne et contemporaine. Le présent numéro thématique de la revue Trivium vise à donner un aperçu de la richesse des deux traditions, plus particulièrement, la recherche qui concerne la figure prophétique en islam.

2 Si le projet PROPHET s'était donné une chronologie précise (les périodes prémoderne et moderne), pour ce numéro le choix thématique porte davantage sur les procédés qui ont conduit, dans le temps, à la sacralisation de la figure prophétique, puis à sa remise en cause, et ceci depuis les origines de l'islam. Le lecteur n'y trouvera donc pas le débat sur l'historicité de la figure de Muhammad. Dès la mise en place du projet, l'équipe avait décidé de ne pas rentrer sur ce terrain de recherche, difficile, bien que prometteur ${ }^{4}$. L'approche choisie est plutôt de montrer comment chaque époque a développé sa propre « représentation » du prophète, et comment, de cette manière, la "présence » du prophète s'est actualisée au sein de sa communauté. Pour ce numéro, un premier groupe d'articles interroge ainsi l'image du prophète dans les corpus 
textuels plus anciens. La constitution de cette image va de pair avec l'établissement de règles et normes sous-jacentes à la transmission et à l'établissement de ce même corpus. Le premier article «Mündliche Thora und Ḥadith im Islam. Überlieferung, Schreibverbot, Redaktion ", de Gregor Schoeler ${ }^{5}$ fut le premier d'une longue série de contributions de l'auteur qui interrogent la relation entre oralité et écriture au début de l'islam ${ }^{6}$. Chronologiquement, ces débats se positionnent certes après la phase initiale de la prédication du prophète et de la mise à l'écrit du Coran. Leur « primauté » réside néanmoins dans le rôle décisif qu'ils ont joué dans l'établissement et la transmission même des matériaux historiques et hagiographiques sur la naissance de l'islam. En discutant les thèses de Goldziher (1850-1921), Schoeler comprend l'interdit du passage à l'écrit (qui ne réussit pas à s'imposer) en relation avec le débat similaire qui avait eu lieu dans le judaïsme, à l'époque de la rédaction de la Mishna et du Talmud, deux corpus transmis par voie orale avant leur passage à l'écrit. Le parallèle avec le cas de l'islam est évident, car la nouvelle religion est également caractérisée par un écart chronologique sensible entre la transmission écrite du Coran et la rédaction des premières collections de traditions prophétiques. Dans le présent article l'auteur propose une réponse nuancée à la question du retard de la fixation dans l'écrit du savoir au début de l'islam. Grâce à l'analyse de la littérature prosopographique, croisée avec l'étude des chaînes de transmission des hadiths, l'auteur suggère qu'en réalité, oralité et écrit ont toujours coexisté. La reconstruction de ce débat nous permet de saisir, pour une époque ancienne, le statut de la parole prophétique dans la communauté naissante, et l'interaction entre différentes modalités de transmission auxquelles les premiers savants ont été confrontés pour fonder leur méthodologie propre de transmission du savoir. Il faut donc garder à l'esprit cette dialectique, parfois tendue, pour comprendre d'où est issu le corpus de notices relatives au prophète étudié dans les articles de Denis Gril ${ }^{7}$ et Samuela Pagani ${ }^{8}$. Cette dernière consacre sa contribution à un épisode particulier de la vie du Prophète : le "récit du choix ", dans lequel « une voix sans nom, ou le Seigneur lui-même, somme le Prophète Muhammad de choisir s'il veut être un prophète roi ou un prophète serviteur ». Comme remarqué par l'auteur, nous sommes en présence ici de deux typologies prophétiques opposées, qui incarnent respectivement le paradigme de la royauté sacralisée (ici encore les parallèles bibliques sont à relever) et celui de la soumission à Dieu. L'auteur résume ainsi le rôle que ce choix a pu signifier pour la communauté des origines :

« La distinction, voire l'opposition, entre le roi et le serviteur est développée dans la tradition, où elle se charge d'importantes connotations politiques et théologiques, relatives en même temps à la nature et à la fonction du Prophète. Le "récit du choix" met en scène la tension entre ces deux composantes de son image sous la forme d'un récit de tentation. Tout comme le récit de la tentation de Jésus au désert [...], il met en abîme un débat sur la nature et la fonction du fondateur qui remonte à la première période de la communauté et ne cesse de se développer au cours de son histoire'."

Cette dichotomie qui traverse la figure prophétique a été, tout au long de l'histoire des sociétés islamiques, productrice d'une tension entre le politique et le spirituel, de leur séparation, ou, au contraire, de leur indissociabilité.

3 Si l'article de Samuela Pagani développe les enjeux et les non-dits d'un seul récit/topos ancien, le texte de Denis Gril s'interroge sur un aspect de la représentation musulmane du prophète amplement négligé dans la recherche académique, à savoir la signification sacrée de son corps. En montrant comment la pensée musulmane met en relation la révélation de la parole divine à la sacralisation de la personne et du corps de son 
récepteur, l'auteur met en évidence le fondement théologique de la vénération des reliques prophétiques, mais permet aussi de comprendre l'attachement de la tradition islamique à la description physique du prophète. La personne du prophète apparaît, aussi bien dans sa dimension spirituelle et corporelle, comme le lieu de rencontre entre le transcendant et l'immanent, et de ce fait comme celui à travers lequel l'espace, le temps, les objets et les personnes sont sacralisés. De cette manière, la lecture en finesse de ces récits fait remonter en surface une préoccupation qui a dû être majeure dans la première communauté, même si les tendances plus puritaines de l'islam prémoderne et moderne ont essayé et tentent toujours d'éradiquer cette présence physique, voire thaumaturgique, du prophète au sein de sa communauté. Notons donc que les premiers traités consacrés au culte des reliques du prophète naissent en tant que réponse apologétique aux attaques des tenants d'un islam dit «puritain », incarné parfaitement par les écrits de Muhammad 'Abd al-Wahhāb étudiés par Riexinger.

Cette profonde relecture des collections canoniques de hadith croisée à un questionnement novateur, permet aux auteurs de faire émerger de ce corpus un ensemble de questions (paradigme de la royauté dans l'antiquité tardive, centralité du corps dans le charisme religieux) dont les termes diffèrent profondément de ceux préconisés par la tradition elle-même. La question de l'établissement d'un modèle étant ainsi posée, les sources islamiques plus anciennes représentent ce qu'une mémoire collective a voulu retenir, voire produire, du prophète, dans un double souci de reconstitution idéale de l'image prophétique et, en même temps, de réponse aux enjeux de l'époque de chaque auteur : fonder/exprimer une image salvatrice du Prophète dans les premiers siècles de l'islam et définir ainsi le rôle de l'islam dans l'histoire du salut humain. Le messianisme ainsi que les motifs sotériologiques sont abordés, puisque la réception ancienne de la figure prophétique s'inscrivait dans le contexte de l'antiquité tardive, marquée par l'attente eschatologique et l'espoir du salut. La deuxième partie du numéro réunit trois articles consacrés à l'évolution du débat théologique autour de la figure prophétique dans l'islam prémoderne et moderne.

L'orientaliste suisse Fritz Meier (1912-1998) ${ }^{10}$, dont l'œuvre fut consacrée en grande partie à l'étude de la mystique islamique, a également laissé une contribution remarquable à l'étude des représentations théologiques du prophète de l'islam. Dans l'article que nous proposons, il analyse en détail un point de prophétologie qui a particulièrement partagé les avis au cours des siècles. Un ensemble de traditions semblent insister sur la présence post-mortem du Prophète. Tout au long des siècles, plusieurs réponses théologiques ont été apportées pour expliquer, soutenir ou réfuter cette thèse, dont la proximité avec le dogme de la résurrection du Christ est frappante et, pour certains, troublante. Meier organise son exposé en deux parties : une première partie consacrée à la reconstruction historique de ce débat, puis une deuxième partie dans laquelle il présente les thèses d'un grand savant du XVe siècle, Suyūṭī (1445-1505). Ces interrogations sont loin d'être marginales au sein de la communauté islamique. Meier explique en conclusion comment ce débat est central dans la définition théologique, d'une part, du wahhābisme, et d'autre part, du soufisme à l'époque moderne. Il anticipe ainsi les deux articles suivants, le premier, une recherche récente de Martin Riexinger ${ }^{11}$ dans laquelle l'auteur effectue une lecture critique de la Vie $d u$ Prophète rédigée par le fondateur du mouvement wahhabite, Muhammad b. 'Abd alWahhāb (1703-1792), et met en lumière les procédés textuels grâce auxquels l'auteur, d'une part, réduit le fondateur de l'islam à une simple figure historique dépourvue de 
tout caractère suprahumain, de l'autre, en établissant des parallèles entre son action politique et celle du Prophète, trouve dans le processus de réécriture pseudo-historique (Riexinger relève de nombreux anachronismes dans le texte) un moyen fort de légitimation de son action et de son idéologie. Riexinger arrive à montrer avec pertinence l'ampleur de la fracture opérée par le wahhābisme dans la pensée sunnite traditionnelle.

6 L'article de Riexinger est très technique. Il présuppose chez le lecteur une connaissance approfondie des épisodes majeurs de la vie du Prophète et de la première communauté. Or cette connaissance n'est peut-être pas toujours nécessaire pour comprendre un point essentiel que l'auteur arrive à mettre en évidence à partir de sa lecture minutieuse de la biographie du Prophète (re)composée par Muhammad b. 'Abd alWahhāb, le père du mouvement wahhabite. En s'appuyant sur des nombreux exemples, l'auteur montre le degré d'interdépendance entre la théologie politique du mouvement et sa vision de l'histoire. Le lien ne paraitrait pas évident si l'auteur n'était arrivé à montrer avec lucidité la décomposition, puis la recomposition de la figure prophète chez Ibn 'Abd al-Wahhāb. Les premiers rédacteurs de ce genre littéraire (la sīra) apparu au IX siècle avaient inclus la figure prophétique, but ultime de la création, dans une vaste histoire universelle. Le MuHtașar de Muhammad b. 'Abd al-Wahhāb revient à cette idée initiale, mais la mission prophétique n'est qu'un épisode dans une longue lutte entre le bien et le mal. C'est donc toute une vision de l'histoire qui est en jeu ici. Le dogme (débattu $)^{12}$ qui affirme qu'il n'y aura pas de nouveau prophète après Muhammad introduit l'humanité dans une nouvelle phase dans laquelle Dieu ne "châtiera " plus (directement) les hommes pour leurs mécréances. C'est maintenant la main de l'homme porteur du message du monothéisme, qui se substitue à l'intervention autrefois directe de Dieu dans l'histoire des hommes pour châtier les communautés ayant mécrues en Ses Envoyés. C'est du moins la vision que le mouvement wahhabite avait de son rôle historique. L'histoire sacrée traditionnelle qui est une histoire du salut, est devenue sous la plume Muhammad b. 'Abd al-Wahhāb une histoire de la perdition de l'humanité, que Riexinger appelle à juste titre Unheilsgeschichte.

7 Plusieurs corollaires sont à relever. La lutte contre la sainteté et le culte des saints s'explique par cette représentation, en soi étonnamment moderne, d'un monde désenchanté. A l'opposé, les formulations classiques et prémodernes du soufisme et du chiisme prévoient une sacralité constante de l'histoire, du fait de l'immanence divine, qui se traduit dans le soufisme sunnite par le rôle des Saints et dans le chiisme par l'héritage des Imams, dont le dernier est toujours vivant ${ }^{13}$. C'est donc une vision de l'histoire que promeut la théologie de Muhammad b. 'Abd al-Wahhāb, qui n'hésite pas à exclure, même du Coran, tout ce qui pourrait contredire son plan d'action militaire. Si dans le Coran Moïse avait encore pu pardonner à son peuple d'être tombé dans l'idolâtrie (l'épisode du Veau d'Or), Muhammad b. 'Abd al-Wahhāb ne tolère plus aucune déviance par rapport à cette nouvelle idée de transcendance divine. On comprend donc l'erreur de vouloir montrer ce mouvement comme «ultratraditionaliste ». L'article de Riexinger montre définitivement que le wahhābisme est en rupture avec des positions qui étaient centrales pour l'identité sunnite classique.

8 L'article de Michel Chodkiewicz ${ }^{14}$ analyse un autre aspect des débats sur le prophète qui ont caractérisé la période moderne. Il s'inscrit dans un questionnement historiographique sur ce que la recherche anglo-saxonne nomme "neo-Sufism », une nouvelle configuration d'un soufisme reformé, qui apparaît au $\mathrm{XVIII}^{\mathrm{e}}$ et XIX ${ }^{\mathrm{e}}$ siècle, 
basé sur un retour à l'exemple prophétique ${ }^{15}$. L'auteur veut démontrer que certains aspects de ce courant ne sont point inédits dans les écrits mystiques et que, bien au contraire, les prémices de cette focalisation sur le prophète dans le parcours de réalisation spirituelle sont plus anciennes que ce que l'on pourrait croire. Mais par-delà du débat historiographique interne aux spécialistes de la matière, il est à relever encore une fois de plus que c'est une certaine vision de «l'historicité » du prophète qui est au centre du débat. La distance temporelle depuis le temps du prophète n'a pas seulement créé une idéalisation de cette époque d'or perdue à jamais (c'est l'ambition de la salafiyya, le retour à la pratique des " pieux ancêtres»). Dans les courants spirituels on observe des nouvelles instances de «mise en présence»/Vergegenwärtigung du prophète: de nouveau rituels, de nouvelles techniques, de nouveaux genres dévotionnels, et aussi, ce que Meier analyse à travers le traité de Suyūṭī, la diffusion de visions à l'état de veille, de rencontres avec un prophète "vivant", qui n'a pas abandonné l'histoire, mais, au contraire, y participe, certes, à un niveau " ésotérique ", réservé à une élite de Saints, mais ceci n'empêche pas aux tenants de cette nouvelle prophétologie qui prône un accès direct à la figure prophétique de s'engager dans l'action politique et de réinvestir donc le présent.

9 Cette introduction veut servir de guide aux lecteurs non spécialistes. Nous ne pouvons pas conclure ces lignes sans remercier l'équipe éditoriale de Trivium pour la qualité des échanges dès notre première rencontre. Nous remercions également les traducteurs ainsi que les relecteurs, plus particulièrement Katrin Heydenreich et Bernd Schwibs pour leur patience, et la pertinence de leurs relectures.

10 Cet ensemble d'articles, mis à la disposition des lecteurs des deux langues, répond à une forte demande de connaissances portant sur la figure fondatrice de l'islam. Le choix de ces articles a aussi été motivé par un souci pédagogique. Dans le cadre du renouveau de l'islamologie en France (une dizaine de postes créés en deux ans) et du développement en Allemagne d'instituts de théologie islamique, la lecture croisée des sources ne pourra que contribuer à rapprocher et à enrichir les questionnements autour de l'islam en Europe.

\section{NOTES}

1. https://prophet.hypotheses.org. Projet dirigé par Rachida Chih (CETOBAC - CNRS) et Stefan Reichmuth (Université de Bochum).

2. Le projet s'articule autour de trois axes principaux :

1. Representations of the Prophet: figuration and controversies

2. Heirs of the Prophet: Legitimation and establishment of Religious Authority and Political Power

3. Prophetic piety between individual and collective experience.

3. Michel Espagne: "Silvestre de Sacy et les orientalistes allemands ", Revue germanique internationale [En ligne], 7|2008, mis en ligne le 15 mai 2011, consulté le 21 mai 2019. URL : http:/ journals.openedition.org/rgi/398; DOI : 10.4000/rgi.398. Voir également Michel Espagne / Nora 
Lafi / Pascale Rabault-Feuerhahn (dir.) : Silvestre de Sacy : le projet européen d'une science orientaliste, Paris : les Éditions du Cerf, 2014.

4. Sur ce sujet, les avancées les plus importantes de la recherche sont quasiment toutes dues à l'œuvre de chercheurs allemands ou de langue allemande, comme Harald Motzki, Gregor Schoeler, Andreas Gorke et Nicolai Sinai. Nous remarquons également le groupe de recherche «Inârah» (Institut zur Erforschung der frühen Islamgeschichte und des Koran, http:// inarah.net), qui publie depuis des années des volumes collectifs en allemand en y déployant la méthode dite «hyper-critique ». La réception de ce collectif de chercheurs reste très limitée à cause de la faible diffusion de l'allemand comme langue scientifique à l'étranger. Leur premier volume, paru en anglais il y a quelques années, commence à éveiller l'attention (et les craintes) de la recherche anglo-saxonne, voir l'article, critique, de Jonathan E. Brockopp : « Islamic Origins and Incidental Normativity ", Journal of the American Academy of Religion, 84/1 (2016), p. 28-43. Le dernier volume a pour objet l'émergence de Muhammad comme figure historique. Plusieurs contributions du volume nient bel et bien son existence factuelle. Cette approche est loin de faire l'unanimité de la communauté scientifique. Voir Die Entstehung einer Weltreligion IV. Mohammed Geschichte oder Mythos?, éd. par Markus Groß et Karl-Heinz Ohlig, Berlin : Hans Schiler Verlag (Inârah - Schriften zur frühen Islamgeschichte und zum Koran, vol. 8), 2017.

5. Gregor Schoeler: "Mündliche Thora und Ḥadīth im Islam. Überlieferung, Schreibverbot, Redaktion ", Der Islam, 66 (1989), p. 213-251.

6. Voir en français du même auteur, Écrire et transmettre dans les débuts de l'islam, Paris : Presses universitaires de France, 2002.

7. Denis Gril : «Le corps du Prophète », in : B. Heyberger / C. Mayeur-Jaouen : Le corps et le sacré en Orient musulman, REMMM, 2006, p. 37-57.

8. Samuela Pagani : «'Roi ou serviteur' ? La tentation du Prophète, ou le choix d'un modèle », in : Nelly Amri / Rachida Chih / Denis Gril (dir.) : Le Prophète en islam : l'instauration d'un modèle et formes de dévotion, Archives des sciences sociales des religions, 178 (2017), p. 43-67.

9. S. Pagani, op. cit., p. 43.

10. http://www.iranicaonline.org/articles/meier-fritz-1.

11. Martin Riexinger : « „Der Islam begann als Fremder, und als Fremder wird er wiederkehren“: Muhammad b. 'Abd al-Wahhabs Prophetenbiographie MuHtașar sïrat ar-rasūl als Programm und Propaganda », Die Welt des Islams, 55 (2015), p. 1-61.

12. Voir le livre de Yousef Sangaré : Le scellement de la prophétie en islam, Paris : Geuthner, 2018.

13. Voir Mohammed Ali Amir-Moezzi: Le Guide divin dans le shi'isme originel. Aux sources de l'ésotérisme en Islam, collection Islam Spirituel, Paris / Lagrasse : Verdier, 1992.

14. Michel Chodkiewicz : "Le modèle prophétique de la sainteté en Islam », Al-Masāq - Journal of the Medieval Mediterranean, 7 (1994), p. 201-226.

15. Voir la synthèse de Albrecht Hofheinz: «Illumination and enlightenment revisited, or: pietism and the roots of islamic modernity », accessible en ligne, https://folk.uio.no/albrech/ Hofheinz_IllumEnlightenment.pdf. Voir également Catherine Mayeur-Jaouen: «Aufklärung islamique", "néo-soufisme", "réformisme musulman", “islamismes" Renouveaux historiographiques de l'histoire de l'islam moderne et contemporain depuis les années 1960 », en ligne : https://ahcesr.hypotheses.org/files/2016/04/AHCESR-nov-2009.pdf. 
INDEX

Mots-clés : islam, prophète, Muhammad

Schlüsselwörter : Islam, Prophet, Muhammad

\section{AUTEURS}

\section{FRANCESCO CHIABOTTI}

Francesco Chiabotti est maître de conférences en islamologie à l'INALCO (Paris) et rattaché au CETOBaC. Pour plus d'informations, voir la notice suivante.

\section{RUGGERO VIMERCATI SANSEVERINO}

Ruggero Vimercati Sanseverino enseigne au Centre de Théologie Islamique de l'Université de Tübingen. Pour plus d'informations, voir la notice suivante. 